\title{
Optimized Control of Vortex Shedding from an Inclined Flat Plate *
}

\author{
Won Tae Joe, ${ }^{\dagger}$ Tim Colonius, ${ }^{\ddagger}$ Douglas G. MacMynowski ${ }^{\S}$ \\ California Institute of Technology, Pasadena, CA, 91125, United States
}

\begin{abstract}
Optimal control theory is combined with the numerical simulation of an incompressible viscous flow to control vortex shedding in order to maximize lift. A two-dimensional flat plate model is considered at a high angle of attack and a Reynolds number of 300. Actuation is provided by unsteady mass injection near the trailing edge and is modeled by a compact body force. The adjoint of the linearized perturbed equations is solved backwards in time to obtain the gradient of the lift to changes in actuation (the jet velocity), and this information is used to iteratively improve the controls. The optimized control waveform is nearly periodic and locked to vortex shedding. We investigate how features of the optimized waveform modify the vortex shedding and lead to higher lift, and compare the results with sinusoidal open- and closed-loop control from a previous study. In the cases investigated, the optimized control is able to achieve higher average lift than the sinusoidal forcing with lower momentum coefficients. In order to obtain a practically implementable control scheme, the optimized waveform is also implemented in a simple closed-loop controller where the control signal is shifted or deformed periodically to adjust to the (instantaneous) frequency of the lift fluctuations. The feedback utilizes a narrowband filter and an Extended Kalman Filter to robustly estimate the phase of vortex shedding and achieve phase-locked, high lift flow states. Finally, the sensitivity of the flow to the phase shift and other features of the optimized waveform are examined.
\end{abstract}

\section{Introduction}

In flapping-wing flight of insects and birds, a leading edge vortex (LEV) has been shown to account for high lift at high angles of attack unanswered by 'quasi-steady' aerodynamic models. ${ }^{1,2}$ The LEV appears to be stable over a significant portion of the stroke, but for a purely translating wing, it is eventually shed and ultimately regular shedding of opposite-sign vortices from the leading and trailing edges occurs. For the translating case, it is of interest to investigate whether active flow control can be used to modify the formation and shedding of vortices in order to obtain unsteady vortical lift comparable to the flapping wing case. Previous work on flow control over an airfoil has used periodic excitation, such as unsteady mass injection or synthetic jets, to show that the oscillatory addition of momentum can delay boundary layer separation and reattach the separated flow, ${ }^{3,4}$ or delay dynamic stall on a rapidly pitching airfoil. ${ }^{5}$ Unsteady actuation was also shown to change the global dynamics of vortex shedding of post-stall flow, leading to higher unsteady lift than the natural shedding. . $^{6} 7$

In this paper, we investigate a simple model of a purely translating flat plate at high angle of attack at a Reynolds number of 300, where strong, periodic vortex shedding occurs. A small amplitude body force intended to mimic oscillatory mass injection is applied near the trailing edge is order to modulate the vortex shedding. In our previous work, ${ }^{8}$ we considered open and closed-loop control utilizing sinusoidal waveforms. It was observed that open-loop forcing can significantly amplify the lift, but that the frequency of oscillation has to be carefully adjusted in order to lock on to the vortex shedding and achieve the highest average lift. For other frequencies, subharmonic limit cycles occur and degrade the performance. It was found that a

\footnotetext{
*Work supported by a Multidisciplinary Research Initiative from the United States Air Force Office of Scientific Research (FA9550-05-1-0369, Program Manager: Dr. Fariba Fahroo).

${ }_{\dagger}^{\dagger}$ Ph.D. Candidate, Mechanical Engineering, Student Member AIAA

${ }^{\ddagger}$ Professor, Mechanical Engineering, Member AIAA

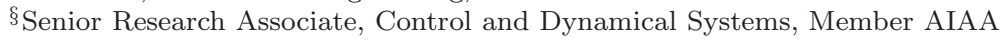


feedback controller designed to tune the phase of actuation to a particular phase of the measured lift was able to achieve lock-on for a wider range of frequencies, and resulted in higher average lift.

In this paper, we consider the same model and investigate the possibility of optimizing the lift using more general (non-sinusoidal) actuation waveforms. We utilize a gradient-based approach that has been used previously in simulations to reduce the turbulent kinetic energy and drag of a turbulent flow in a plane channel, ${ }^{9}$ or to reduce free-shear flow noise. ${ }^{10}$ Given the DNS for a particular actuator waveform, we solve the adjoint of the perturbed linearized equations backward in time to determine the sensitivity of the lift to the actuator input, and subsequently use this information to iteratively improve control.

This computed optimal control requires knowledge of the full flow state and therefore is not practical for real-time control. Instead, we use a period of the optimal waveform together with the previously developed phase-locking feedback strategy in order to provide a robust and practical approach to giving near-optimal performance.

In the next section, we present the simulation methodology and the actuation scheme. In order to provide the comparison between performance of the optimized control signal to that of sinusoidal control signal, open- and closed-loop control from the previous work is briefly reviewed in section III. Once the objective of our control is defined, we formulate an adjoint-based optimization in section IV.A, and compare the results to the previous sinusoidal forcing in section IV.B. In section V, we design a feedback algorithm where the optimized waveform is shifted or deformed periodically to adjust to the output frequency of the flow. We show that the feedback controller achieves as high lift as the optimization, and can be started from any phase of the natural shedding cycle. Finally, we investigate the sensitivity of the lift to the phase shift and other features of the optimized waveform.

\section{Governing Equations and Numerical Method}

Simulations of flow over a two-dimensional flat plate at $R e=300$ and an angle of attack of $40^{\circ}$ are performed with the immersed boundary projection method combined with a vorticity-streamfunction multidomain technique. ${ }^{11,12}$ We model the actuation as a point body force smeared over a few grid points with its strength defined by its velocity as $U_{\text {jet }}$. This method solves the incompressible viscous flow equations, presented here in operator form by (1). The control is implemented as a velocity boundary conditions $\phi(x, t)$ applied at the actuation points $\mathscr{C}$ shown in figure 1 . In this paper, control is a function only of time, and $\phi(x, t)=\phi(t)=U_{\text {jet }}(t)$, which is the prescribed velocity at the actuation point. The operator form of our flow equations is then

$$
\mathscr{N}(\mathbf{q})=\mathbf{F} \phi(t)
$$

where $\mathbf{q}$ is a vector of flow variables,

$$
\mathbf{q}=\left[\begin{array}{ll}
\gamma & \tilde{f}
\end{array}\right]^{\mathrm{T}} \text { and } \mathbf{F}=\left[\begin{array}{ll}
F_{\gamma} & F_{\tilde{f}}
\end{array}\right]^{\mathrm{T}} .
$$

$\gamma$ is the discrete circulation and $\tilde{f}=\left[\begin{array}{ll}\tilde{f}_{x} & \tilde{f}_{y}\end{array}\right]$ is a vector of surface forces on the Lagrangian body points applied to satisfy the no-slip condition for a stationary body points or the prescribed velocity for the actuation points. The vector $\mathbf{F}$ allocates the control action and has a single non-zero entry that corresponds to the actuator location associated with the appropriate element of the surface force, $\tilde{F}$.

This method is capable of solving for incompressible flows over an arbitrary-shaped body in motion and deformation. Here we employ this method with a stationary, rigid flat plate. In what follows, all velocities and length scales are nondimensionalized by the freestream velocity and the chord, $U_{\infty}$ and $c$, respectively.

The numerical method utilizes a series of overlapping uniform Cartesian grids of differing resolution. ${ }^{12}$ The finest grid, encompassing the body, is comprised of a rectangular domain exending to $[-1,4] \mathrm{X}[-1.5,1.5]$ in the streamwise $(x)$ and vertical $(y)$ directions with a uniform grid spacing of 0.02 units. The constant time step was 0.004 . The coarsest grid extended to $[-8,32] \mathrm{X}[-12,12]$. The boundary condition at the outermost grid was that the streamfunction corresponding to the difference between the full velocity and a uniform free stream was zero. Selected cases were run on finer grids and with larger extents to demonstrate convergence and independence to far-field boundary conditions.

The lift and drag coefficient on the flat plate is defined by

$$
C_{\mathrm{L}}=\frac{F_{y}}{\frac{1}{2} \rho U_{\infty}^{2} c} \quad \text { and } \quad C_{\mathrm{D}}=\frac{F_{x}}{\frac{1}{2} \rho U_{\infty}{ }^{2} c}
$$


where $F_{y}$ is the lift on the plate and $\rho$ is the freestream density of the fluid. Since the force obtained is normal to the plate and $F_{y}$ is only the vertical component of the normal force, the increase of the normal force increases both the lift and drag. As the angle of attack increases, the drag component of the normal force is increased while the lift component is reduced. For high angles of attack, this might result in decrease of the lift-to-drag ratio even in the presence of lift enhancement. However, for the purpose of demonstrating the control algorithm to achieve high lift, we will pay closer attention to the lift component of the normal force, $C_{\mathrm{L}}$.

In defining the momentum injection added by the forcing, the width of the actuator is estimated as the grid spacing, $\Delta x$. The momentum coefficient, defined in Eq. (4), is the ratio between the momentum injected by the forcing and that of the freestream.

$$
C_{\mu}=\frac{\rho{\overline{U_{\text {jet }}(t)}}^{2} \Delta x}{\frac{1}{2} \rho U_{\infty}^{2} c} \quad C_{\mu}^{\prime}=\frac{\rho\left\langle U_{\text {jet }}(t)\right\rangle^{2} \Delta x}{\frac{1}{2} \rho U_{\infty}^{2} c}
$$

The values of $C_{\mu} C_{\mu}^{\prime}$ reported are based on the average and the root mean square of control input, $\overline{U_{\text {jet }}(t)}$ and $\left\langle U_{\text {jet }}(t)\right\rangle$, respectively and the width of the actuator, $\Delta x=0.02$.

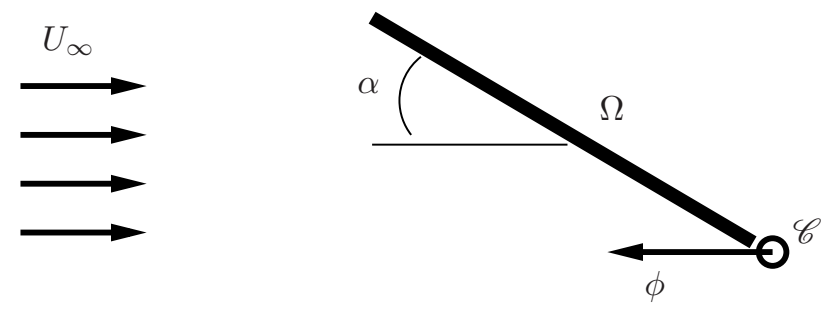

Figure 1. Schematic of upstream actuation at the trailing-edge.

\section{Sinusoidal Feedback Control}

In previous work on unsteady actuation, ${ }^{8}$ we considered sinusoidal forcing to increase lift. The control was applied as a nondimensional jet velocity, $\phi=\overline{U_{\text {jet }}}+U_{\text {jet }}^{\prime} \sin \left(\omega_{\mathrm{f}} t\right)$, where $\overline{U_{\text {jet }}}=U_{\text {jet }}^{\prime}$ thus $C_{\mu}=C_{\mu}^{\prime}$. Since the goal was to maximize lift from shedding of the coherent vortex structures rather than the suppression of shedding or separation, the frequency, $\omega_{\mathrm{f}}$, was initially chosen to be the natural shedding frequency for each angle-of-attack $\alpha$. The flow was phase locked to the actuation for $\alpha \leq 15^{\circ}$. However, for $\alpha \geq 20^{\circ}$, a subharmonic resonance was excited and over each period of forcing there was a phase shift between the forcing and lift signals. Certain phase shifts resulted in very high period-averaged lifts. It is interesting to note that, by using a Reynolds-averaged two-dimensional computation of a turbulent flow over an airfoil at post-stall angles of attack, Wu et al. ${ }^{7}$ also observed that the highest-lift vortex shedding cycle was not in perfect frequency lock-in with the actuation, but the subharmonic resonance was also excited.

With the goal of obtaining phase-locked forced limit cycles with the maximum average lift, we designed a feedback algorithm that demodulates the lift signal, applies a low-pass filter, adds a phase shift, and finally remodulates, in order to output a sinusoidal forcing with a specified phase shift relative to the lift signal. Figure 2 shows the time history of the lift coefficient $C_{\mathrm{L}}$ and the control signal $\phi(t)=U_{\text {jet }}(t)$ for open- and closed-loop control at $\alpha=40^{\circ}$. By adjusting its forcing corresponding to the change of output frequency from the flow, the feedback phase lock the flow to the actuation at a distinct phase shift that corresponds to the highest average lift at this $C_{\mu}=0.01$. As a result, the feedback achieved a stable limit cycle with an average lift and drag coefficients of $\overline{C_{\mathrm{L}}}=2.25$ and $\overline{C_{\mathrm{D}}}=1.83$, corresponding to the average lift-to-drag ratio of $\overline{C_{L} / C_{\mathrm{D}}}=1.20$. Compared to baseline flow $\left(\overline{C_{\mathrm{L}}}=1.35, \overline{C_{\mathrm{D}}}=1.20\right.$, and $\left.\overline{C_{\mathrm{L}} / C_{\mathrm{D}}}=1.104\right)$, feedback resulted in more than $65 \%$ increase in average lift. 

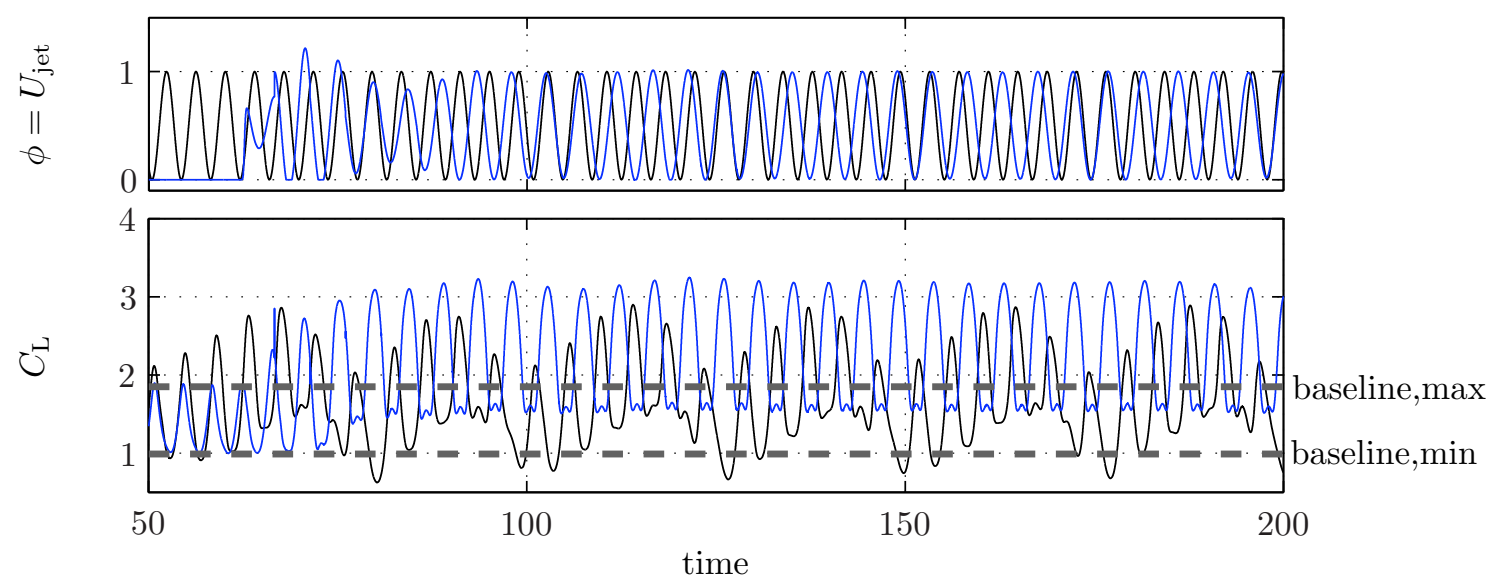

Figure 2. Sinusoidal forcing with $C_{\mu}=C_{\mu}^{\prime}=0.01$ at $\alpha=40^{\circ}$ : Open-loop forcing at natural shedding frequency is plotted in black and closed-loop forcing plotted in blue. Maximum and minimum lift of baseline (- - -) case is shown as a reference.

\section{Optimization}

\section{IV.A. Formulation}

The feedback strategy from the previous study optimizes the phase of the control relative to the lift, but always uses a sinusoidal waveform. However, continuous sinusoidal forcing could be adding circulation when it is unnecessary, or undesirable. Here we employ an adjoint-based optimization in order to find the waveform that maximizes the lift for a given actuation amplitude. We compute the optimal control over a time horizon, using the receding-horizon approach. ${ }^{9}$ The procedure is simlar to previous studies ${ }^{9,10}$ and is only outlined briefly here.

To maximize lift, we define a cost functional to be minimized

$$
\mathscr{J}=-\int_{t_{0}}^{t_{1}} \int_{\Omega} \tilde{f}_{y}^{2}(\phi(t), x, t) d x d t+C_{w} \int_{t_{0}}^{t_{1}} \int_{\mathscr{C}} \phi^{2}(t) d x d t
$$

where $t_{0}$ and $t_{1}$ are the start and end times of the optimization horizon and $\Omega$ is surface of the body (see figure 1). $\phi$ is the control input, in this case $\phi(t)=U_{\text {jet }}(t)$. The first term is the total squared lift over the optimization horizon. The second term penalizes the actuator amplitude in order to keep $C_{\mu}$ to a value commensurate with the open-loop control discussed previously. The control weight, $C_{w}$, is determined by trial and error as discussed below.

At each iteration of the optimization, we modify the controls according to

$$
\phi^{k+1}=\phi^{k}+r * g\left(\phi^{k}\right),
$$

where $g(\phi)$ is the gradient of the cost function with respect to the controls, and $r$ is the generalized distance determined iteratively (using Brent's line minimization) to minimize the cost function. $g(\phi)$ is found by solving

$$
g(\phi)=F_{\tilde{f}} \cdot \tilde{f}^{*}+2 C_{w} \phi,
$$

where $\tilde{f}^{*}$ are the force unknowns in the linearized adjoint equations ${ }^{13}$

$$
\mathscr{N}^{*}(\mathbf{q}) \mathbf{q}^{*}=\mathbf{F}^{*} \text {. }
$$

Here $\mathbf{q}^{*}$ are the adjoint variables (discrete circulations and forces) and $\mathbf{F}^{*}$ is given by

$$
\mathbf{F}^{*}=\left[\begin{array}{ll}
F_{\gamma}^{*} & F_{\tilde{f}}^{*}
\end{array}\right]^{\mathrm{T}}=\left[\begin{array}{ll}
0 & 2 \tilde{f}_{y}
\end{array}\right]^{\mathrm{T}} .
$$

The adjoint operator requires the full flow field from the (forward) Navier-Stokes simulation at every time step. However, in order to save memory, we saved the flow solution only every few time steps and used a linear interpolation in time. Several test cases were done with a different number of time steps skipped, 
including a case where the solution was saved at every time step, and no significant differences were noted between them.

All optimizations used zero control $(\phi=0)$ for the first iteration $(k=1)$ on each optimization horizon. At each iteration, we required roughly ten full Navier-Stokes simulation to perform the line minimization (to find $r$ ).

Optimization was done over a horizon $T=\left[\begin{array}{ll}t_{0} & t_{1}\end{array}\right]$, where the horizon, $T$, is long enough to overcome transient effects, but limited by the computational effort to perform all the required iterations and to tune the control weight. We found for this problem that after about two periods the controls converged to an approximately periodic signal with each period corresponding to a vortex shedding cycle. A horizon of 6 periods gave the results presented below, and tests showed that the results were not very sensitive as the horizon was varied from about 5 to 8 periods. Once the iteration of the optimization converges, the control near the end of each optimization horizon (transient of adjoint simulation) is discarded and the optimization is begun anew. This process is depicted in figure 3.

The control weight, $C_{w}$, was chosen by trial and error to be 0.3 . This gives the results shown in the next section where $C_{\mu}$ was about two times lower than that used for the sinusoidal forcing, but achieved comparable lift.

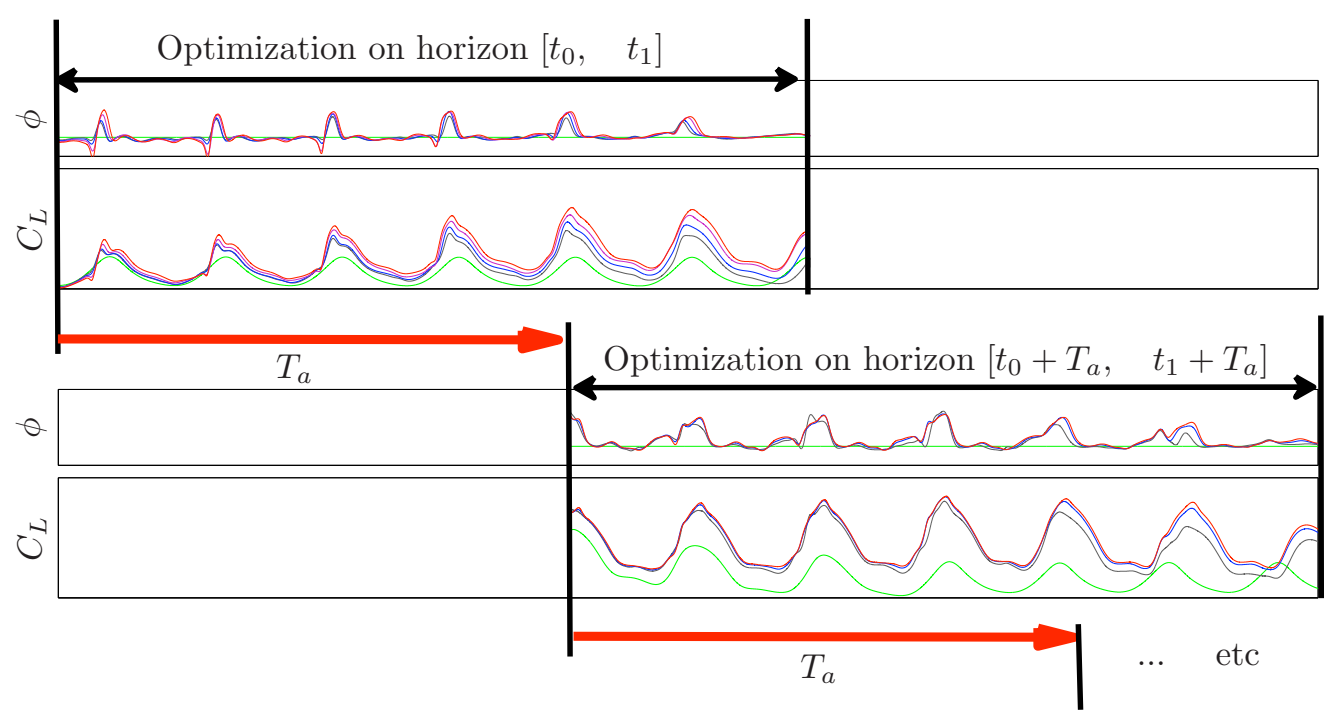

Figure 3. Schematic of receding-horizon predictive control. First the optimization of controls are performed on horizon $\left[t_{0}, t_{1}\right]$. Each iteration of optimization gives the update on control. Once the convergence of the control on the optimization is achieved, the flow is 'advanced' some portion $T_{a}$ of the period $T$, and controls near the end of the optimization horizon are discarded and the optimization is begun anew on horizon $\left[t_{0}+T_{a}, t_{1}+T_{a}\right]$.

\section{IV.B. Results of optimization}

Figure 4 compares a few periods of the converged optimal control signal $\left(U_{\text {jet }}(t)\right)$ and the resulting lift coefficient. The results are plotted against the previous closed-loop controlled case with a sinusoidal waveform. Recall from section III that this compensator phase locked the flow at a limit cycle associated with the highest average lift at a given $C_{\mu}$. The optimized control is not sinusoidal, but rather composed of two distinct pulses per shedding cycle. The larger, later pulse is roughly in phase with the maximum lift. In figure 5, we overlay the vorticity contours at the minimum and maximum lift for the baseline and sinusoidal forcing and compare vorticity contours for the optimized control to the sinusoidal forcing in figure 6. Maximum lift is found when the LEV is brought down to the suction side of the plate as it grows in strength. The lift decreases as the new vortex structure of the opposite sign is formed at the trailing edge. Minimum lift occurs as this TEV pushes up the LEV sitting on the plate, and finally halts its growth causing it to pinch-off and shed into the wake.

For both sinusoidal and optimized control, the primary effect of actuation is to create extra vorticity which is fed into the TEV as the vortices are alternately being formed and shed. ${ }^{8}$ After the first local minimum lift, as the new LEV is being formed, both waveforms start to feed extra circulation at the trailing edge, leading to more definite pinch off of the LEV at the following global minimum lift as shown in figure 5(a). The 
magnitude of actuation increases as the growing LEV (lift is increasing) is pulled down by the growing TEV, and finally reaches its peak near the maximum lift. The corresponding TEV is strengthened and caused to shed from the trailing edge, thereby allowing the LEV to grow larger. This results in more vertically elongated TEV that induces stronger downwash near the trailing edge, causing the LEV to sit closer to the plate, leading to higher lift compared to the baseline.

The flow field for the optimized control does not look very different from the sinusoidal forcing. However, in the optimized control, the short pause between the two pulses slows down the growth of the TEV momentarily. This separates the TEV into two structures combined by a thin vortex sheet as shown in figure $6(\mathrm{~b})$. The effect of the dip between the two pulses will be investigated further with the feedback control in section $\mathrm{V}$.

The most distinct feature of the optimized control compared to sinusoidal forcing is the gradual increase in $\phi$ during the most of the cycle followed by a more rapid decrease after its peak. A gradual addition of circulation alters the formation of the TEV such that it interferes minimally with the natural formation of LEV and only acts as a downwash to push the LEV closer to the plate. Immediately after the maximum lift, the forcing is turned off sharply. This phase of the shedding cycle is where the optimized control achieves similar magnitude of lift with minimal control input compared to the sinusoidal control. Since the shedding of the LEV is probably unavoidable in two-dimensional flow (no spanwise flux of vorticity in $z$-direction) after the maximum lift has been achieved, letting it shed naturally may be the most energy efficient. For the periods shown in figure 4, optimized control resulted in $\overline{C_{L, \text { ave }}}=2.50$ and $\overline{C_{D}}=2.06\left(\overline{C_{L} / C_{D}}=1.20\right)$ with $C_{\mu}=0.005$ and $C_{\mu}^{\prime}=0.010$.

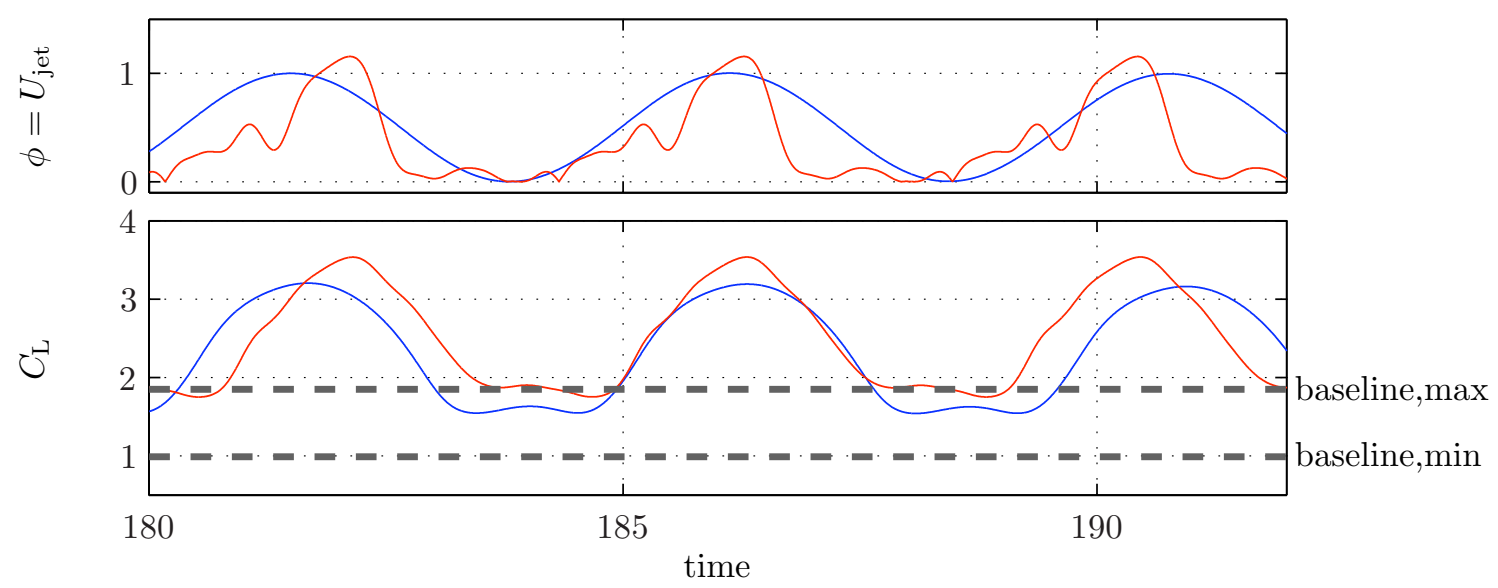

Figure 4. Comparison of optimized control $(-)$ with closed-loop sinusoidal forcing from figure $2(-)$. Maximum and minimum lift of baseline (- - ) case is shown as a reference.

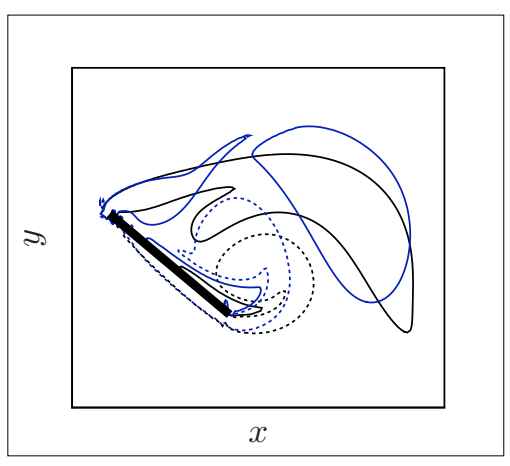

(a) Minimum $C_{L}$

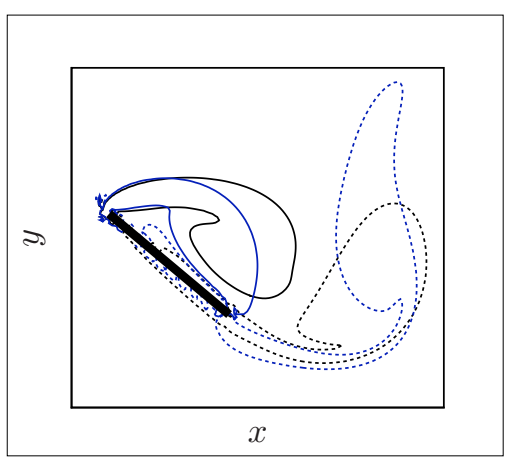

(b) Maximum $C_{L}$

Figure 5. Vorticity contour at the (a) minimum and (b) maximum lift for baseline (black) and sinusoidal actuation (blue). Dashed and solid lines represent counterclockwise and clockwise vorticity. 


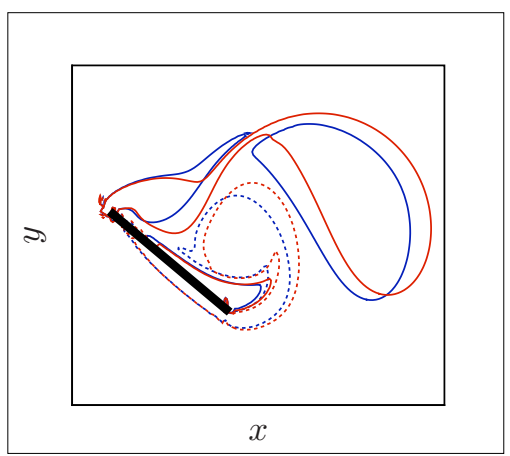

(a) Minimum $C_{L}$

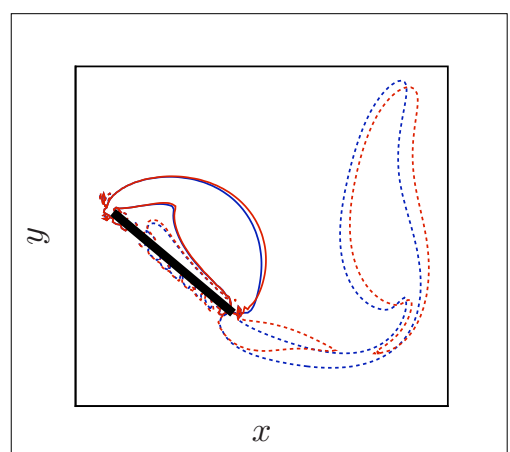

(b) Maximum $C_{L}$

Figure 6. Vorticity contour at the (a) minimum and (b) maximum lift for sinusoidal actuation (blue) and optimized actuation (red). Dashed and solid lines represent counterclockwise and clockwise vorticity.

\section{Feedback}

In this section, we design a practically implementable feedback algorithm to achieve phase lock between the lift and the optimal control waveform deduced from the adjoint-based algorithm in the previous section. While it is straightforward to extract a single period of the optimal waveform, the performance can be significantly degraded if this is applied to the plate in open loop. Depending on the precise state of the flow upon initiation of forcing, the flow fails to lock onto the optimal waveform or locks on with a different phase than the optimal controller. Moreover, initial transients and subharmonic resonances further degrade the performance.

In order to design the controller, we revisit and extend an idea implemented in our previous study of sinusoidal waveforms. In that paper, a feedback compensator was designed that demodulated the lift signal, applied a low-pass filter, shifted the phase by a specific amount, and remodulated the signal in order to produce a sinusoidal output locked by a specified lag to the lift signal.

Implementation of this controller for the optimal waveform is a more complex task, since the waveform now comprises multiple harmonics of the fundamental frequency. For example, we may write

$$
\begin{aligned}
\phi_{\text {optimal }}(t) & =A_{0}+\sum_{k}^{N_{k}}\left[A_{k} \cos (k \omega t)+B_{k} \sin (k \omega t)\right] \\
& =A_{0}+\sum_{k}^{N_{k}}\left[A_{k} \cos (k \theta(t))+B_{k} \sin (k \theta(t))\right],
\end{aligned}
$$

where $N_{k}$ is the number of harmonics retained and $\omega$ is the fundamental frequency of the optimal waveform. We used $N_{k}=10$ which provided a reasonable representation (less than $5 \%$ deviation from the original optimized waveform).

In order to implement this optimal waveform with a consistent phase difference between each of the harmonics, instantaneous phase information of the lift signal is required. The frequency of the lift signal is tracked with an Extended Kalman Filter (EKF) to estimate the phase, $\theta(t)$ for use in Eq. 10. To improve the EKF phase estimate, narrowband filter is first used on the lift cycle to obtain a more nearly sinusoidal signal as input to the EKF. The EKF frequency estimate is then used to tune the filter to avoid introducing phase lag. The overall feedback algorithm is illustrated in figure 7.

First, we perform a narrowband filtering of the lift according to

$$
\begin{aligned}
\omega_{\mathrm{i}} & =2 \pi / T_{\mathrm{i}}, \\
a_{1}(t) & =\frac{2}{T_{\mathrm{i}}} \int_{t-T_{\mathrm{i}}}^{t} C_{\mathrm{L}}\left(t^{\prime}\right) \cos \left(\omega_{\mathrm{i}} t^{\prime}\right) d t^{\prime}, \\
b_{1}(t) & =\frac{2}{T_{\mathrm{i}}} \int_{t-T_{\mathrm{i}}}^{t} C_{\mathrm{L}}\left(t^{\prime}\right) \sin \left(\omega_{\mathrm{i}} t^{\prime}\right) d t^{\prime}, \\
y(t) & =a_{1}(t) \cos \left(\omega_{\mathrm{i}} t\right)+b_{1}(t) \sin \left(\omega_{\mathrm{i}} t\right) .
\end{aligned}
$$


The filtered lift, $y(t)$ retains the dominant frequency, initially estimated as $\omega_{i}$, and filters out higher harmonics. Next, $y(t)$ is modeled as a pure sinusoid

$$
\begin{aligned}
& \hat{y}(t)=\hat{a} \sin (\hat{\theta}(t)), \\
& \hat{\theta}(t)=\hat{\omega}_{\mathrm{o}} t,
\end{aligned}
$$

where $\hat{\theta}$ is estimated with the EKF; values for noise processes are chosen in the EKF so that the algorithm converges in a few cycles. Our implementation of the EKF follows closely the description in Tadmor ${ }^{14}$ and Patoor et al. ${ }^{15}$

When computing $y(t)$, the initial estimate for $\omega_{i}$ is updated with the estimate $\hat{\omega}_{o}$, the frequency estimated by the EKF, and we write

$$
\phi_{\text {optimal }}(t)=A_{0}+\sum_{k}^{N_{k}}\left[A_{k} \cos \left(k\left(\hat{\theta}(t)-\theta_{\text {desired }}(t)\right)\right)+B_{k} \sin \left(k\left(\hat{\theta}(t)-\theta_{\text {desired }}(t)\right)\right)\right],
$$

where $\theta_{\text {desired }}$ is an additional (specified) phase shift relative to the lift signal.

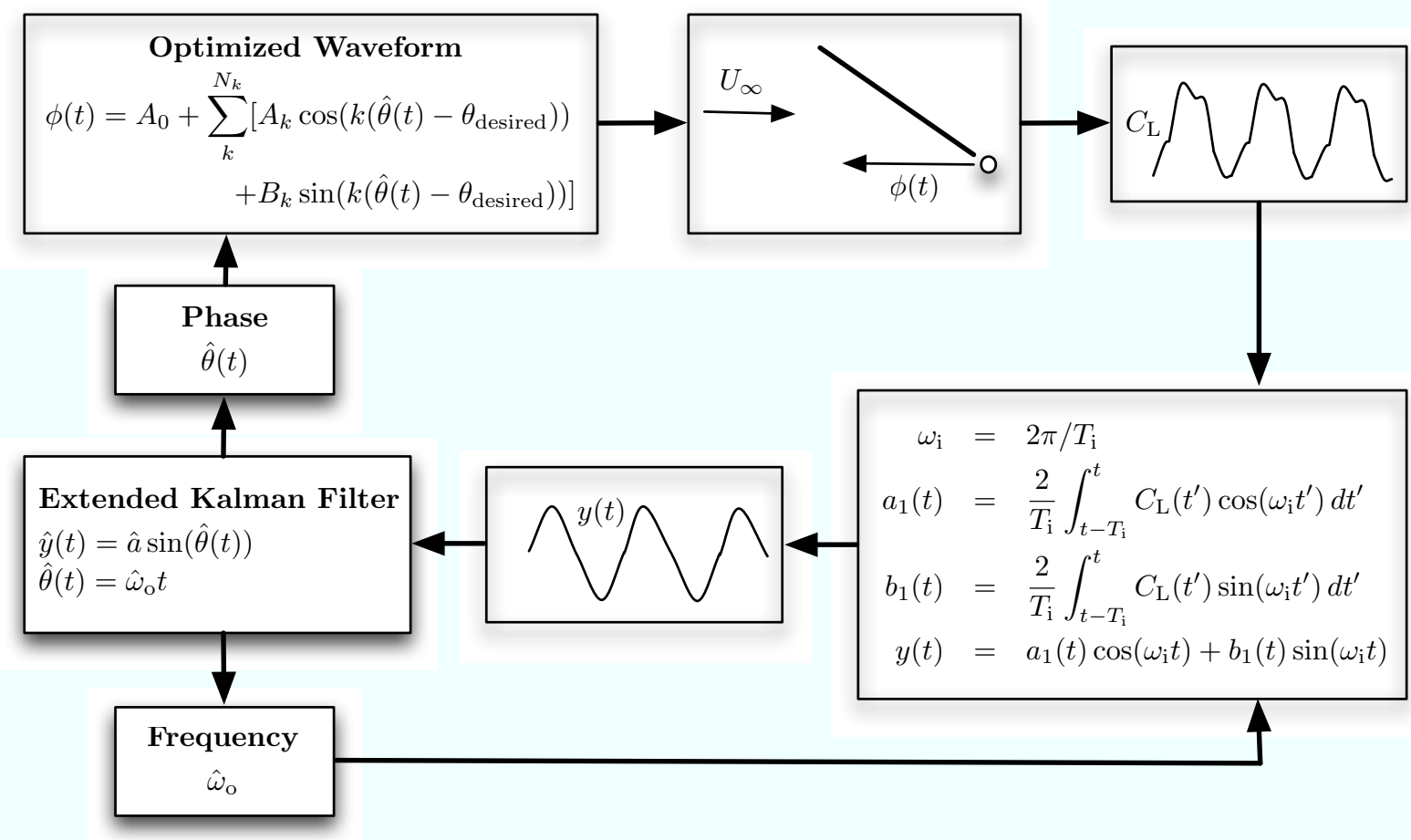

Figure 7. Schematic of feedback.

As shown in figure 8, feedback control of the optimized waveform is able to reproduce the high-lift limit cycle from the previous section, but starting from an arbitrary phase of the baseline limit cycle. The feedback system converges to something very close to the previous solution after 4 to 5 periods, and is indistinguishable after about 10 cycles.

The feedback controller now allows us to phase-lock an essentially arbitrary waveform, and we can utilize this fact to investigate which features of the optimized waveform are critical to high lift. In figure 9, we demonstrate the effect of smoothing the optimal waveform by retaining fewer harmonics in the Fourier expansion. Using $N_{k}=4$, for example, smoothes out the dip between the two highest maxima, but has little impact on the lift achieved. This indicates that, during this phase of the shedding cycle, the sensitivity of the first term (lift-maximizing term) in Eq. 5 to the change in $\phi$ is small compared to the second term 
(control-penalizing term). The short pause between the two pulses may be just an energy-saving feature of the optimal control.

Figure 10 investigates the sensitivity of the lift performance of the phase-locked limit cycles to the changes in the phase shift, $\theta_{\text {desired }}$ with the optimal control $\left(N_{k}=10\right)$. Feedback is able to phase lock the flow at any desired phase shift after $3 \sim 5$ periods over a wide range of $\theta_{\text {desired. }}$. Due to pulse-like feature of the optimal waveform, the lift is quite sensitive to changes in the phase shift, with the average lift dropping below the maximum lift of the baseline with $20^{\circ}$ phase changes. Because the optimized waveform rapidly decreases immediately after its peak, forcing with the peak prior to the maximum lift (at negative phase shift) impacts the lift significantly. Also, positive phase shift penalizes the lift performance since the magnitude of $g(\phi)$ (sensitivity of the cost functional, Eq. 5 to changes in control, $\phi$ ) is small during the lift-decreasing phase; thus, control is not as effective. As phase shift approaches $\pm 180^{\circ}$ (out of phase), the forced flow results in the average lift similar to that of an unforced flow.
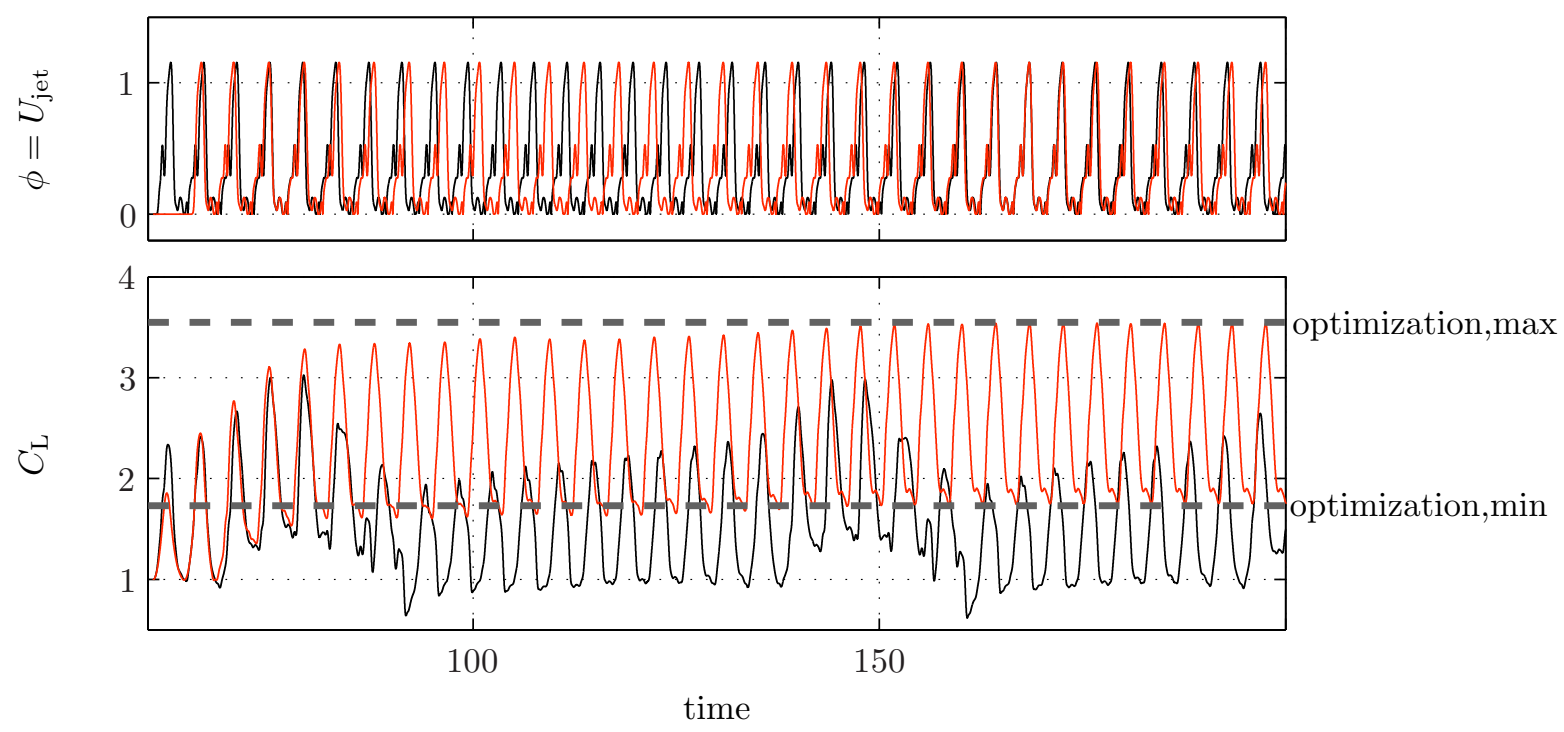

Figure 8. Comparison between open-loop control case $(-)$ and the feedback control case $(-)$ with optimized waveform $\left(N_{k}=10\right)$.

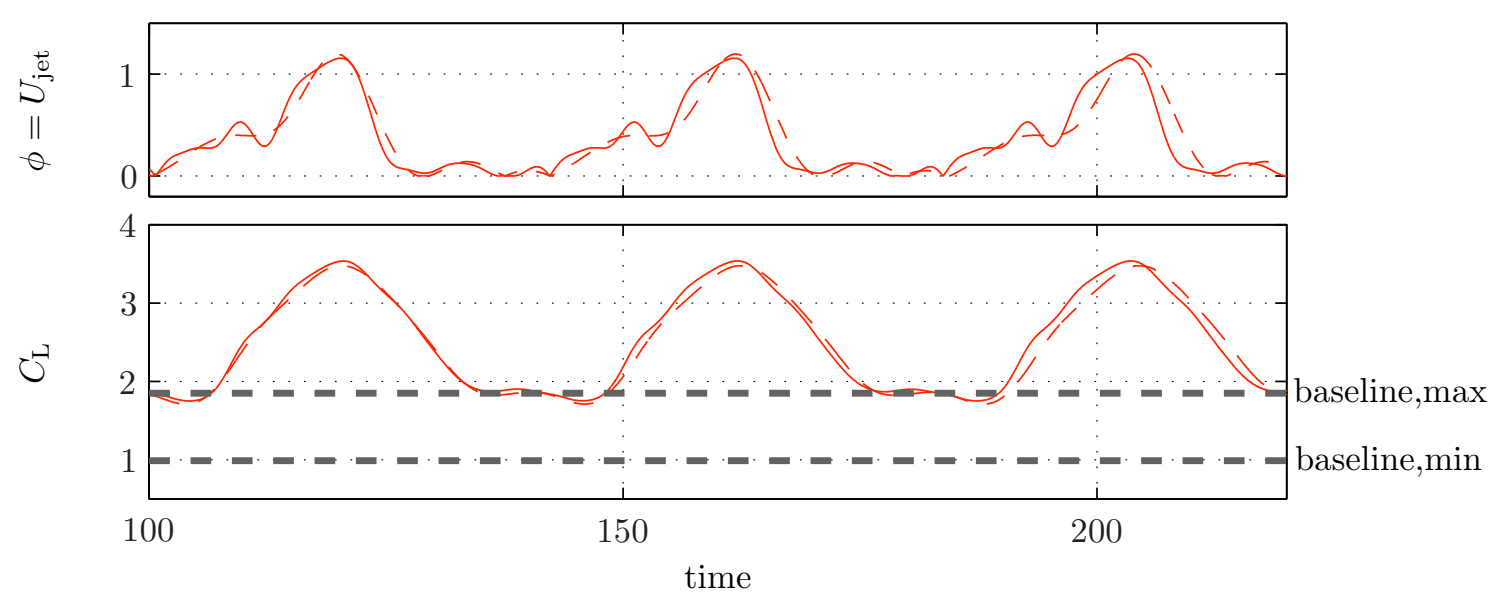

Figure 9. Comparison between feedback control cases with optimized waveform: $N_{k}=10(-)$ and $N_{k}=4(---)$. 


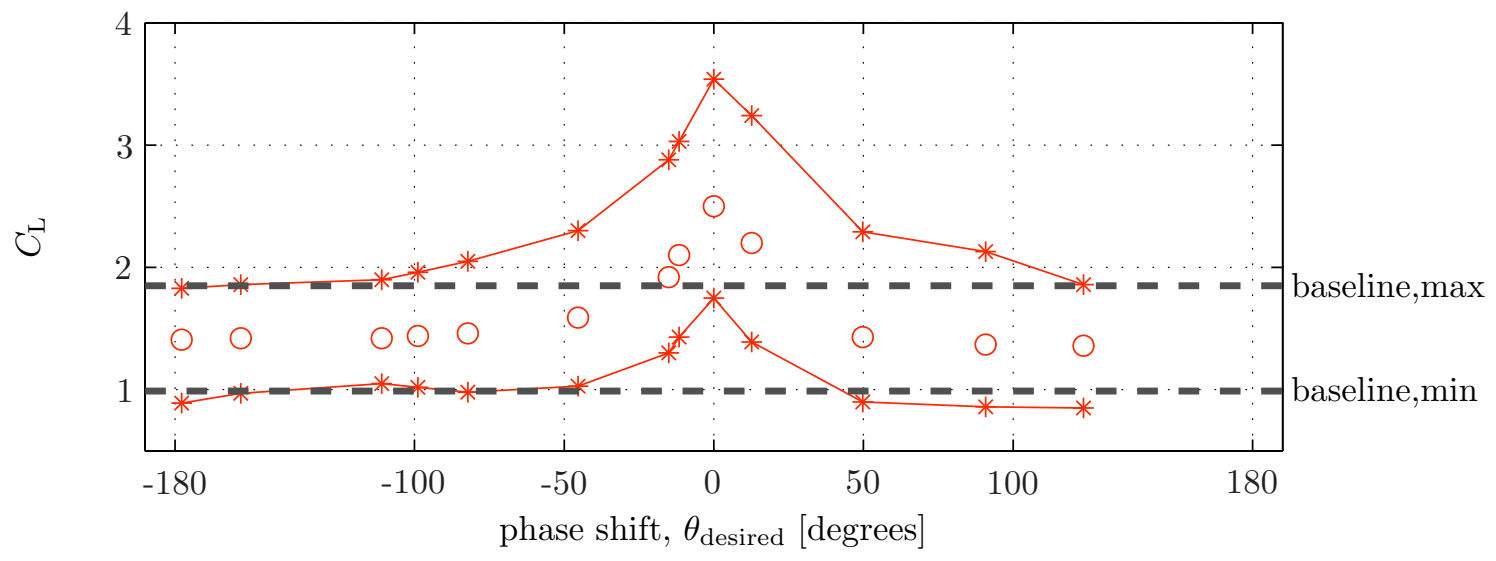

Figure 10. Maximum and minimum lift $(*)$ and average lift $(O)$ of phase-locked limit cycles at different phase shift with optimized waveform $\left(N_{k}=10\right)$. Maximum and minimum lift of baseline (- - ) case is shown as a reference.

\section{Summary}

A gradient-based (adjoint) approach was applied in a receding-horizon setting to optimize the control waveform in order to maximize the lift on a two-dimensional airfoil at $\alpha=40^{\circ}$ and $R e=300$. The optimized control waveform is not sinusoidal, but rather is pulse-like, with each period composed of two distinct pulses (a primary, as well as a smaller earlier pulse). It is interesting to note that pulsatile waveforms, with pulse durations much shorter than the convective time scale, have also been shown to be effective in open-loop forcing in separation control. ${ }^{16}$ The most distinct feature of the optimal control is a gradual increase in the forcing $\phi$ during most of the cycle, followed by a more rapid decrease after its peak. This minimal control effort after the maximum lift, combined with the short pause between the two pulses provides more energyefficient control than sinusoidal forcing. As a result, the optimal control achieves comparable lift with 2 times lower $C_{\mu}$ value $\left(\overline{C_{\mathrm{L}}}=2.50\right)$ with $\left.C_{\mu}=0.005\right)$ as the sinusoidal forcing case $\left(\overline{C_{\mathrm{L}}}=2.25\right)$ with $\left.C_{\mu}=0.010\right)$.

Optimal control provides a periodic control waveform. However, if applied in open loop, the flow fails to phase lock onto the optimal waveform, degrading the lift performance. We designed a feedback algorithm to obtain phase-locked limit cycles. Using a Fourier representation of the optimized waveform, $\phi_{\text {optimal }}$, the control parameterizes the waveform in terms of its phase $\theta(t)$, allowing the feedback to march along $\phi_{\text {optimal }}$ with consistent phase difference between each of its modes. The control consists of the following steps : 1 . A narrowband filter is used on the lift cycle to obtain a more nearly sinusoidal signal. 2. The filtered lift signal is used as input to frequency tracking Extended Kalman Filter (EKF) to estimate the phase, $\hat{\theta}(t)$ of the lift signal. 3. The EKF frequency estimate is used to tune the filter to avoid introducing phase lag. 4. Finally, the phase estimate $\hat{\theta}(t)$ from EKF is used to march along $\phi_{\text {optimal }}$.

Feedback control of the optimized waveform was able to reproduce the high-lift limit cycle from the optimization, but starting from an arbitrary phase of the baseline limit cycle. Also, it allowed us to phase lock an essentially arbitrary waveform, thus enabling us to investigate the sensitivity of the flow to the phase shift and other features of the optimized waveform. By using fewer harmonics in the Fourier expansion of the optimized waveform, we demonstrated that smoothing the dip between the two pulses has little impact on the lift performance; this characteristic is more of an energy-saving feature. We also showed that the phase-locked limit cycle with optimized waveform was sensitive to changes in the phase shift, $\theta_{\text {desired }}$ from $0^{\circ}$. The sharp decrease in lift performance with negative phase shift is due to the steep drop in the optimized waveform after its peak. The lift penalty with positive phase shift indicates that the forcing is less effective after the maximum lift has occurred.

Understanding the optimal waveform, combined with the phase-locking feedback strategy, provides a robust and practical implementation of control to give near-optimal performance. In future investigations, we will explore different parameters, such as the location of actuation or differing control goals (minimize drag, minimize oscillation, etc.). 


\section{References}

${ }^{1}$ Dickinson, M. H. and Gotz, K. G., "Unsteady aerodynamic performance of model wings at low Reynolds-numbers," Journal of Experimental Biology, Vol. 174, 1993, pp. 45-64.

${ }^{2}$ Ellington, C. P., vandenBerg, C., Willmott, A. P., and Thomas, A. L. R., "Leading-edge vortices in insect flight," Nature, Vol. 384, No. 6610, 1996, pp. 626-630.

${ }^{3}$ Glezer, A. and Amitay, M., "Synthetic jets," Annual Review of Fluid Mechanics, Vol. 34, 2002, pp. 503-529.

${ }^{4}$ Greenblatt, D. and Wygnanski, I. J., "The control of flow separation by periodic excitation," Progress in Aerospace Sciences, Vol. 36, No. 7, 2000, pp. 487-545.

${ }^{5}$ Magill, J., Bachmann, M., Rixon, G., and McManus, K., "Dynamic stall control using a model-based observer," Journal of Aircraft, Vol. 40, No. 2, 2003, pp. 355-362.

${ }^{6}$ Rullan, J. M., Vlachos, P. P., Telionis, D. P., and Zeiger, M. D., "Post-stall flow control of sharp-edged wings via unsteady blowing," Journal of Aircraft, Vol. 43, No. 6, 2006, pp. 1738-1746.

${ }^{7} \mathrm{Wu}$, J. Z., Lu, X. Y., Denny, A. G., Fan, M., and Wu, J. M., "Post-stall flow control on an airfoil by local unsteady forcing," Journal of Fluid Mechanics, Vol. 371, 1998, pp. 21-58.

${ }^{8}$ Joe, W. T., Taira, K., Colonius, T., MacMynowski, D. G., and Tadmor, G., "Closed-loop control of vortex shedding on a two-dimensional flat-plate airfoil at a low Reynolds number," AIAA Paper, 2008-634.

${ }^{9}$ Bewley, T. R., Moin, P., and Temam, R., "DNS-based predictive control of turbulence: an optimal benchmark for feedback algorithms," Journal of Fluid Mechanics, Vol. 447, 2001, pp. 179-225.

${ }^{10}$ Wei, M. J. and Freund, J. B., "A noise-controlled free shear flow," Journal of Fluid Mechanics, Vol. 546, 2006, pp. 123152 .

${ }^{11}$ Taira, K. and Colonius, T., "The immersed boundary method: A projection approach," Journal of Computational Physics, Vol. 225, No. 2, 2007, pp. 2118-2137.

${ }^{12}$ Colonius, T. and Taira, K., "A fast immersed boundary method using a nullspace approach and multi-domain far-field boundary conditions," Computer Methods in Applied Mechanics and Engineering, Vol. 197, No. 25-28, 2008, pp. 2131-2146.

${ }^{13}$ Ahuja, S. and Rowley, C. W., "Low-dimensional models for feedback stabilization of unstable steady states," AIAA Paper, 2008-553.

${ }^{14}$ Tadmor, G., "Observers and feedback control for a rotating vortex pair," Ieee Transactions on Control Systems Technology, Vol. 12, No. 1, 2004, pp. 36-51.

${ }^{15}$ Pastoor, M., Henning, L., Noack, B. R., King, R., and Tadmor, G., "Feedback shear layer control for bluff body drag reduction," Journal of Fluid Mechanics, Vol. 608, 2008, pp. 161-196.

${ }^{16}$ Amitay, M. and Glezer, A., "Flow transients induced on a 2D airfoil by pulse-modulated actuation," Experiments in Fluids, Vol. 40, No. 2, 2006, pp. 329-331. 